\author{
MARTA SMYKAŁA \\ Uniwersytet Rzeszowski \\ smykala@intertele.pl
}

\title{
Fremdheit übersetzen. Erfahrungen aus einem übersetzerischen Projekt einer studentischen Arbeitsgemeinschaft
}

\author{
Translating foreignness. \\ Experience from a translation project carried \\ out by a students' project group
}

\begin{abstract}
The article partially sums up the results of a translating project realized by a students' science club. The aim of the project was to translate into German an interview with the caretaker of the synagogue in Łańcut. The interview deals with the history of the building and the history of the former Jewish community in Łańcut. Particularly problematic in the translation process were terms originating from Yiddish or Hebrew: realia, cultural artefacts, religious terms and some other exoticisms. Another barrier was connected with the ability to approach the text from the receiver's perspective. The article discusses those and other difficulties involved in the process of translation and some possible ways of solving them.
\end{abstract}

KEYWORDS: translation, translating foreignness, cultural specific, translating realia, Jewish culture and religion.

Dieser Beitrag ist Herrn Wolfgang Maloszek, spiritus movens des Vorhabens, und Mitgliedern der studentischen Arbeitsgemeinschaft Hieronymus gewidmet, als Dank für ihr Engagement.

\section{AUSGANGSLAGE}

Die studentische Arbeitsgemeinschaft „Hieronymus“, die in den Jahren 2011-2015 am Institut für Germanistik der Universität Rzeszów verschiedene Aktivitäten unternahm, entstand auf Initiative einer Gruppe von Germa- 
nistikstudierenden, die neben Vertiefung ihres theoretischen Wissens aus dem Bereich der Translationswissenschaft auch noch ihr translatorisches Können ständig auf die Probe stellen wollten. Im Rahmen dieser „übersetzungspraktischen Übungen“ pflegten es die angehenden Übersetzer, jedes Jahr eine andere regionale Sehenswürdigkeit zu besichtigen und das vom Reiseleiter Gesagte ins Deutsche zu dolmetschen. Vor einem solchen Einsatz gab es eine längere Vorbereitungsphase, die sowohl in Gruppentreffen als auch im Rahmen individueller Arbeit (zu Hause) stattfand. Als Vorbereitung kamen verschiedene Aktivitäten in Frage: Analyse von Paralleltexten (in polnischer und deutscher Sprache), gezielte Vorbereitung auf die Besichtigung einer bestimmten Sehenswürdigkeit (Lektüre einschlägiger Literatur), eine vorherige Besichtigung der Sehenswürdigkeit, Konsultationen mit Experten u. a.

Im akademischen Jahr 2013/2014 wurde die von außen eher unauffällige Synagoge in Łańcut zu einem Objekt des Interesses der Studenten. Dies hatte einige Gründe: Objekte jüdischer Kultur / Architektur wecken besonderes Interesse deutschsprachiger Touristen und die Synagoge in Łańcut ist neben dem Schloss Łańcut die zweitwichtigste touristische Attraktion dieser Stadt für deutsche Gruppen. Abgesehen davon ist sie auch eine der schönsten Synagogen in Polen. Darüber hinaus ist das Karpatenvorland eine Region, in der Juden kurz vor dem Ausbruch des Zweiten Weltkriegs etwa 10\% der gesamten Bevölkerung und in manchen Städten der Region beinahe 50\% der Einwohner ausmachten (vgl. Potocki 2004). Die Region war ein kultureller Schmelztiegel, in dem Juden die drittgrößte Bevölkerungsgruppe, nach der polnischen und ukrainischen, darstellten. Trotzdem ist das Jüdische heute eine eher unbekannte Welt für die Bewohner des Karpatenvorlands. Für die Projektteilnehmer bedeutete das eine Fülle von neuen Eindrücken, Informationen, Bezeichnungen.

Als Grundlage für die Vorbereitung auf einen potenziellen DolmetschEinsatz diente den Studierenden ein umfangreiches Interview, das online auf der Homepage des Forums Polnischer Juden (Forum Żydów Polskich, FŻP) abzurufen war (und ist) ${ }^{1}$. Nach einer kurzen Absprache mit dem Interviewten wie auch dem Journalisten, d.h. dem Autor des Textes, welcher seinerseits im Namen der Arbeitsgemeinschaft eine Absprache ebenfalls mit dem FŻP hielt, sowie der Stiftung zum Schutz Jüdischen Erbes gingen die Projektteilnehmer ans Werk. Eines war dabei von vorneherein klar: Das Vorhaben braucht eine muttersprachliche Unterstützung. Herr Wolfgang Maloszek, ein in Rzeszów lebender deutscher Lehrer (Germanist) sagte dem

${ }^{1}$ Vgl. http://www.fzp.net.pl/spoleczenstwo/szojmer-z-lancuta (letzter Zugriff am 11.08.2016). 
Vorhaben zu. Bis zu diesem Zeitpunkt waren Eifer und Ehrgeiz kaum zu dämpfen. Nun begann aber die harte Arbeit, deren Ergebnisse unter http://www.fzp.net.pl/spoleczenstwo/szojmer-z-lancuta-0 abzurufen sind.

\section{ZIELSETZUNG}

In diesem Beitrag möchte ich einerseits auf die im Laufe des Prozesses aufgetauchten translatorischen Schwierigkeiten und gewonnenen Erkenntnisse, andererseits auch auf die den ganzen Prozess begleitenden sowie die sich aus ihm ergebenden didaktischen Reflexionen hinweisen. Mein besonderes Augenmerk gilt allerdings denjenigen Textstellen, die unvertraute Ausdrücke enthalten und die ich hier allgemein als kulturspezifisch (exotisch) bezeichne. Damit meine ich Realien, Eigennamen, Fremdwörter und Fremdbezeichnungen, die im AS-Text vorkommen, aus dem Hebräischen bzw. Jiddischen stammen, sich auf die jüdische Kultur und Religion beziehen und "irgendwie" in den ZS-Text übertragen werden mussten. Das Gefühl der Fremderfahrung ist übrigens sowohl bei der Lektüre als auch bei der Übersetzung des Textes sehr stark, aber genau dieses Fremde ist es, welches die Eigenartigkeit (Spezifik) des Textes in weiten Teilen ausmacht ${ }^{2}$. Deswegen war eine der Intentionen der Projektteilnehmer, diese besondere Tonalität des AS-Textes in der Übersetzung zu erhalten. Das bedeutete, möglichst viele exotische Bezeichnungen des AS-Textes im ZS-Text stehen zu lassen. Die nachstehend angeführten Beispiele konkreter translatorischer Probleme und Lösungen können als Ausgangspunkt für allgemeine Bemerkungen dienen.

Auf „lernerspezifische Probleme“ (vgl. Nord 2011: 116), sprich die Beseitigung offensichtlicher Fehler, wird hier nicht eingegangen. Im Weiteren wird auf translatorische lernerunabhängige Probleme fokussiert.

\section{TRANSLATORISCHE STOLPERSTEINE}

Diese hatten mehrere Ursachen, von denen m. E. die drei wichtigsten sind:

- Unkenntnis der Sache,

- Spezifik des Ausgangstextes,

- mangelnde übersetzerische Erfahrung.

2 Der Problematik wie man das Jüdische in der Übersetzung handhaben kann, wurde u. a. ein ganzes Heft von Przekładaniec (29/2014) gewidmet: vgl. Przekład żydowski. Żydowskość w przektadzie. 
Wie bereits gesagt, wurden lernerspezifische Probleme aus weiteren Überlegungen ausgeschlossen. Darauf rekurriert die Bezeichnung „mangelnde übersetzerische Erfahrung". Sie kommen in diesem Beitrag nicht zur Geltung, dennoch halte ich es für begründet, auf diesen Umstand deutlich hinzuweisen, denn Problematisches wird bei mangelnder Erfahrung noch problematischer.

\subsection{Kulturspezifik oder Unkenntnis der Sache}

Die Sprache einer untergegangenen Kultur zu übersetzten ist die eine Schwierigkeit. Oft muss man sich ihre frühere Existenz erst einmal bewusst machen. Die jüdische Kultur in Galizien ${ }^{3}$ ist ein unabänderliches Faktum. Ihre Unkenntnis ist ebenfalls ein Faktum, an dem sich allerdings etwas ändern lässt. Die Erscheinungsformen einer Kultur sind Artefakte verschiedener Art (vgl. Burszta 2008). In dem zu übersetzenden Text kommen viele Bezeichnungen von sprachlichen und nicht-sprachlichen Artefakten vor, die, und das ist eine weitere Schwierigkeit, in eine ebenfalls fremde Kultur, nämlich die deutsche, übersetzt werden mussten. In einem als kultureller Transfer aufgefassten Übersetzungsprozess geht es mehrheitlich um Vermittlung zwischen zwei verschiedenen Kulturen, von denen zumindest eine dem Übersetzer vertraut ist. Im besprochenen Fall kam noch eine dritte - jüdische, den Projektteilnehmern wenig bekannte Kultur dazu, was den Übersetzungsprozess wesentlich erschwerte. Es galt, diese Kultur ein Stück weit kennenzulernen und zu erforschen: Wie die Juden gefeiert und gelebt haben, welche Feste es gab und wie sie zelebriert wurden, welche Gegenstände man im Alltag (und an Feiertagen) benutzte, wie die Einrichtung der Synagoge aussah und hieß und welche Bedeutung all das für die Kultur als solche hatte. Aber verständlicherweise war es auch notwendig, sich mit dem Judentum und seinen Ausprägungen, insbesondere dem Chassidismus ${ }^{4}$, sowie dem sozialen Leben einer jüdischen

\footnotetext{
${ }^{3}$ Gemeint sind der südöstliche Teil Polens (Westgalizien) sowie eine Landschaft in der Westukraine (Ostgalizien).

${ }^{4}$ Chassidismus - eine religiöse Bewegung innerhalb des Judentums, entstanden im 18. Jahrhundert, die - laut Duden - eine lebendige Frömmigkeit anstrebt (vgl. http://www.duden. de/rechtschreibung/Chassidismus). Die Heimat des Chassidismus ist Mittel- und Osteuropa. Die Synagoge in Łańcut ist u. a. dank großer Chassidim z. B. Jaakow Jizchak Horowitz und Naftali Cwi Horowitz (Naftali von Ropschütz) bekannt. Galizien war neben Podolien und Wolynien (Ukraine) eine Wiege des Chassidismus.
} 
Gemeinschaft ${ }^{5}$ oder auch den sozialen Rollen beider Geschlechter ${ }^{6}$ auseinanderzusetzen.

Die Unkenntnis der jüdischen Kultur betraf (und betrifft) allerdings nicht nur die studentischen Übersetzer. Es ist nicht schwer, sich vorzustellen, dass auch das (Vor-)Wissen des deutschsprachigen Zieladressaten in Bezug auf die behandelten Inhalte bescheiden ausfallen mag. Dazu käme noch die Unkenntnis der polnischen / galizischen Gegebenheiten. Gemeint sind etwa polnische geographische Namen von Orten, z. B. Ropczyce, Dzikowiec sowie von Personen und adeligen Geschlechtern, z. B. Potoccy, Lubomirscy und ihre Bedeutung für die polnische Kultur.

Expandieren bot sich als eine der einzusetzenden Übersetzungsstrategien an (vgl. Kautz 2000: 66), wobei Kautz unter Strategie ein „zielorientiertes kognitives Verhalten [...], einen mehr oder minder bewussten Plan zur Lösung der konkreten Übersetzungsprobleme“" versteht. Der Übersetzer hat das vom Autor / Sprecher Implizierte und beim muttersprachlichen Empfänger als Vorwissen Vorausgesetzte zu verbalisieren (vgl. Kautz 2000: 325). Anders gesagt, beim Übersetzen ist immer ein Perspektivenwechsel notwendig. In der Fachliteratur ist hier oft von der "Ausrichtung an der zielkulturellen Situation" (vgl. Nord 1999: 146) die Rede. In Bezug auf das gegenständliche Projekt war sogar ein mehrfacher Perspektivenwechsel unumgänglich. Es galt zusätzlich, die unterschiedlichen historisch bedingten Verhältnisse zwischen Polen, Juden und Deutschen jeweils zueinander zu berücksichtigen.

\subsection{Perspektive und Perspektivenwechsel (Adressatenbezug)}

Die zu übersetzende Welt mit den Augen des Rezipienten zu sehen, benötigt immer einen Perspektivenwechsel und hohes Einfühlungsvermögen. Der Übersetzer geht als Sprach- und Kulturmittler mehreren Rollen nach: Er ist Leser und Empfänger des zu übersetzenden Textes, das bedeutet, dass er den jeweiligen Text einmal als Translator, einmal als AS-Leser und einmal als ZS-Adressat wahrnimmt. In dem hier beschriebenen Projekt war wohl

\footnotetext{
${ }^{5}$ In der Synagoge sind z.B. Matzewas von jüdischen Friedhöfen aus Łańcut ausgestellt. Somit erfährt man bei der Besichtigung auch einiges über die mit dem Begräbnis zusammenhängenden Bräuche (Grabinschriften, Symbole und ihre Bedeutung). Als Vorentlastung diente uns u. a. ein gemeinsames Ansehen des polnisch-deutschen Dokumentarfilms Po-lin. Okruchy pamięci [Po-lin. Spuren der Erinnerung] (2008) von Jolanta Dylewska über Juden in der Zwischenkriegszeit in Polen, mit anschließender Diskussion.

${ }^{6}$ Etwa Wissen um „Kompetenzen“ der Geschlechter: sehr vereinfachend und verallgemeinernd gesagt: Die Frau als diejenige, die das Zuhause in ihrer Obhut hatte und der Mann als derjenige, der die Tora studierte und zur Synagoge ging.
} 
die Fähigkeit dieses multiplen Perspektivenwechsels, die eigentlich eine übersetzerische Grundkompetenz ist bzw. sein sollte, alles andere als selbstverständlich vorhanden.

Die jungen Übersetzer zerbrachen sich zwar den Kopf darüber, wie sie z.B. das Adjektiv łańcucki/Lancuter im Deutschen wiedergeben können (im Originaltext u. a. in der Bezeichnung Icyk Lancuter oder łańcucki święty)" sahen sich aber oft nicht gezwungen, dem Zielempfänger nahezulegen, dass das Adjektiv łańcucki von Łańcut, einer Kleinstadt in Südostpolen, abgeleitet ist. In der studentischen Rohfassung stand daher: „Hier in Łańcut wurde er Icyk Lancuter oder der Landshuter Heilige genannt" und es bedurfte einer eifrigen Diskussion, bis man sich darauf einigte, dass:

- geographische Selbstverständlichkeiten (Toponyme) für einen Fremden (Zielempfänger) nicht unbedingt selbstverständlich sind; daher ist ein zusätzlicher erläuternder Hinweis, was damit gemeint ist, oft notwendig,

- geographische Namen sowie die davon abgeleiteten Adjektive im gesamten Text einheitlich zu verwenden sind,

- man die polnischen Bezeichnungen beibehält: Łańcut und nicht etwa Landshut - zumal Landshut eher auf den deutschen Ort referiert.

Auch die jedem Polen bekannten Geschlechternamen, wie z.B. die zuvor erwähnten Potockis, verlangen zwecks besserer Leserfreundlichkeit eine zusätzliche Erläuterung, dass es sich eben um ein adeliges polnisches Geschlecht handelt. Auch dafür mussten die Projektteilnehmer oftmals sensibilisiert werden $^{8}$, was auf ihre mangelnde übersetzerische Erfahrung zurückzuführen war und für einen erfahrenen Übersetzer eher ein Gemeinplatz ist.

Das expandierende Verfahren bezog sich nicht nur auf geographische Bezeichnungen und polnische Adelsnamen. Erläuterungen bedurften ganz besonders die vielen fremden Bezeichnungen und Begriffe aus dem Hebräischen und Jiddischen.

\subsection{Babiniec, Bereschit und Tewje Milchmann. Umgang mit Exotischem und übersetzungsrelevantes Recherchieren}

Im Ausgangstext waren es vor allem Bezeichnungen jüdischer Realien, die allen Projektteilnehmern besondere Schwierigkeiten bereiteten. Sitz-

\footnotetext{
7 Vgl. die Endfassung: „Hier in Łańcut wurde er Icyk Lancuter oder der Heilige von Łańcut genannt."

${ }^{8}$ Im untersuchten Text kommen die jeweiligen Namen immer mit vorangestellten Adelstiteln, etwa Graf oder Herzog, vor, daher ergab sich eine zusätzliche Erläuterung als überflüssig. Der Hinweis aber, die Perspektive des Adressaten nie aus den Augen zu verlieren, war dennoch wichtig, zumal die Übersetzung eine Vorentlastung vor einem potenziellen Dolmetsch-Einsatz war.
} 
fleisch und Geduld waren notwendig, aber nicht ausreichend, um zu den gewünschten Lösungen und Entscheidungen zu kommen. Um geeignete Äquivalente zu finden, war vor allem das mühselige Stöbern im Internet notwendig. Das Internet stellte sich letztendlich als das aktuellste und umfangreichste Medium heraus. Verschiedene Druckquellen, sprich gedruckte Parallel- und Hintergrundtexte (oft einschlägige Literatur), halten dem Internet nicht stand. Gedruckte Quellen sind zwar meistens glaubwürdiger, die Internetquellen dagegen aktueller und vielfältiger. Aus dem wenig strukturierten und ungefilterten Angebot des Internets (vgl. Kautz 2000: 106) wählen zu können ist allerdings auch eine Fertigkeit, die man zwar als Übersetzer im Griff haben, die aber zunächst gelernt bzw. geübt werden muss. Dabei ist die Frage nach der Glaubwürdigkeit der Quellen von besonderer Bedeutung. In den das Projekt begleitenden Diskussionen kam oft zum Vorschein, dass eine richtige Einschätzung der im Internet gefundenen möglichen Äquivalente für angehende Übersetzer problematisch ist und dass man sie oft für die Glaubwürdigkeit der Quellenseiten sensibilisieren muss, vor allem dafür, ob die Webseite, die sie gerade als Grundlage für ihre translatorische Entscheidung nehmen, nicht etwa selbst eine Übersetzung ist. Wichtig ist auch, wer oder was die Seite redigiert und für die Inhalte steht, ob es eine glaub- und vertrauenswürdige Einrichtung, Organisation, Person ist.

Es ist noch anzumerken, dass auch ein aufwendiges Recherchieren im Internet nicht immer zu eindeutigen Ergebnissen führte. Als Beispiel sei hier das Wort babiniec genannt. Die meisten Studierenden nehmen heute keine gedruckten Wörterbücher mehr in Anspruch. Sie benutzen kostenlose zweisprachige Online-Wörterbücher (hauptsächlich Pons oder dep.pl, depl.dict.cc). Das populärste einsprachige Online-Wörterbuch ist duden.de. Sein „Aufschlagen" ist aber weniger häufig als die Inanspruchnahme zweisprachiger Online-Wörterbücher. Es kann also nicht verwundern, dass die Projektteilnehmer das polnische Wort babiniec zunächst als Weiberschar / Frauenschar übersetzt haben. Eine solche Entsprechung (allerdings im landläufigen Sinne von einer Schar, d.h. „einer größeren Anzahl“ von Frauen) gibt Pons mit Anmerkung pot (umgangssprachlich) an. Etwas tiefer auf dem Bildschirm, weil es im Teil OpenDict enthalten ist (von Benutzern veröffentlichte Stichworte), ist noch eine Entsprechung angeführt, nämlich Frauengebetsraum. Diese Entsprechung wurde von den Studierenden zunächst nicht wahrgenommen und blieb unberücksichtigt. Wohlgemerkt, der Wortanfang Weiberist ziemlich verlockend, wenn auch irreführend. Er konnte vermuten lassen, dass es sich tatsächlich um etwas (z.B. einen Raum) für Weiber (Pl. baby) handele. Das Wort Weiberschar ist zwar weder im Online-Duden noch in der gedruckten Fassung dieses Wörterbuchs verzeichnet, wohl aber im digitali- 
sierten Deutschen Wörterbuch von Jacob Grimm und Wilhelm Grimm, das online zugänglich ist ${ }^{9}$. Seine Bedeutung hat jedoch mit einer Mechisa (aus dem Hebräischen "Trennung" im Sinne der Trennungsvorrichtung) bzw. Weiberschul (aus dem Jiddischen „Räumlichkeiten für Frauen“, „Frauensynagoge“) nichts gemeinsam. In diesem Fall war die Suche nach einem Äquivalent nicht unbedingt mit einem zweisprachigen Wörterbuch zu beginnen, zumal babiniec in vielen Wörterbüchern nicht erfasst ist. Deswegen galt als Faustregel bei der Übersetzung: Sobald festgestellt wird, dass ein Wort keine Entsprechung in einem (umfangreichen) zweisprachigen Wörterbuch hat, sollte dies von jedem Projektteilnehmer als Warnsignal wahrgenommen werden. In Bezug auf das Lexem babiniec war ein gezieltes Suchen nach einer Bezeichnung für einen Raum für Frauen in einer Synagoge in der Zielsprache effizienter und sicherer. Die Suche konnte erfolgen, indem man z.B. die Bauweise von Synagogen oder die Geschlechtertrennung in orthodoxen Synagogen eingegeben und studiert hat. Bei etwas Glück konnte man dann ziemlich schnell auf die Bezeichnungen Mechisa und Weiberschul stoßen, aber in den meisten Fällen werden die Räumlichkeiten für Frauen als Frauenemporen, Frauengalerien oder Frauengebetsräume bezeichnet. Das Wort Weiberschul bedeutet eigentlich "Frauensynagoge", denn Schul ist das jiddische Wort für "Synagoge“ (das Wort Synagoge kommt ursprünglich aus dem Griechischen). Weiberschul liegt dem polnischen babiniec als lexikalische Entsprechung am nächsten, es scheint aber im Deutschen nicht so verbreitet zu sein wie das polnische babiniec. Dem Zieladressaten und einer besseren Verständlichkeit des ZS-Textes zuliebe wurde den allgemein verständlichen Bezeichnungen Frauengalerie bzw. Frauenempore, Frauengebetsraum Vorrang gegeben. Im Polnischen bezieht sich das Wort babiniec auch auf einen „Vorraum für Frauen in orthodoxen Kirchen“. Wohl deswegen wird es manchmal auch als Kirchenhalle ${ }^{10}$ übersetzt. Im ZS-Text wäre aber eine Bezeichnung mit Kirche- irreführend, denn Kirche evoziert Assoziationen mit dem Christentum.

Die meisten exotischen Bezeichnungen, die im Text vorkommen, hängen mehr oder weniger mit der jüdischen Religion zusammen. Es gibt auch Ausnahmen: Tewje mleczarz wurde unproblematisch identifiziert und auf Tewje Milchmann umgetauft [wobei er eher mit dem Lied If I Were a Rich Man als mit dem Film Jewisons aus dem Jahre 1971 Anatevka (Fiddler on the Roof) assoziiert wurde].

Allen Projektteilnehmern war auch das Wort Bereszit / Bereschit nicht bekannt. Es schien die Bezeichnung einer Realie zu sein. Nachforschungen

\footnotetext{
${ }_{9}$ Vgl. http:// woerterbuchnetz.de/DWB/?sigle=DWB\&mode=Gliederung\&hitlist=\&pattern list=\&lemid=GW12531 (Zugriff am 17.08.2016).

${ }^{10}$ Vgl. https://de.glosbe.com/pl/de/babiniec (Zugriff am 18.08.2016).
} 
ergaben jedoch, dass es sich um die hebräische Bezeichnung für das 1. Buch Mose aus dem Pentateuch - hebräisch Tora - handelt. Die Bezeichnung war fremd, nicht aber das bezeichnete Objekt. Das Buch ist unter dem griechischen Namen Genesis bzw. dem Polnischen Księga Rodzaju bekannt. Nichtsdestoweniger wäre eine Übersetzung mit Genesis wenn nicht falsch (das gleiche Denotat - das 1. Buch Mose), so doch hinsichtlich konnotativer Bedeutung und potenzieller Assoziationen, die das Wort beim Adressaten hervorruft, eher fehl am Platze (nicht mit Juden und Judentum und jüdischer Welt verbunden). Die Beibehaltung der hebräischen Eigenbezeichnung, die für den Leser eine Fremdbezeichnung ist und zur besonderen Färbung des Textes beiträgt, und ihre gleichzeitige Explikation schienen deswegen als Lösung am besten.

Der Frage danach, warum babiniec nicht mit Weiberschul, sondern mit Frauenempore und Bereszit mit Bereschit, dem 1. Buch Mose, übersetzt wurde, ist damit vielleicht nicht ganz erschöpfend beantwortet. Hieran zeigt sich aber exemplarisch, dass man es beim Übersetzen mit bestimmten Spielräumen zu tun hat, deren Handhabung letztendlich zu den Kompetenzen eines Übersetzers gehört und als seine Freiheit bezeichnet werden kann. Auf diese Weise kann auch nachvollzogen werden, warum ein AS-Text mehrere ZS-Texte ergeben kann.

\subsection{Umgang mit Exotischem - Übersetzungsstrategien und Übersetzungsverfahren}

Zu Übersetzung von Realia ${ }^{11}$ bzw. kulturspezifischen, landeskonventionellen Elementen ${ }^{12}$ gibt es eine reiche einschlägige Literatur. Markstein (1999: 288) versteht „Realie als Element des Alltags, der Geschichte, der Kultur, der Politik u. drgl. eines bestimmten Volkes, Landes, Ortes, die keine Entsprechung bei anderen Völkern, in anderen Ländern, an anderen Orten hat." Sie betont noch eine Eigenschaft dieser sprachlichen Elemente: Sie „sind Identitätsträger eines nationalen/ethnischen Gebildes, einer nationalen/ethnischen Kultur" (ebenda) und als solche sind sie, ihrer Natur nach, emotiv, d.h. stilistisch nicht neutral und tragen damit zur Steigerung der Expressivität eines Textes bei.

Ganz eindeutig ist die Abgrenzung des Begriffs Realie allerdings nicht. Ist die oben erwähnte Bezeichnung Bereschit eine Realienbezeichnung? Der

11 Vgl. u. a. Markstein (1999: 288).

12 Vgl. u. a. Koller (1997: 232). 
Begriff 1. Buch Mose funktioniert auch in polnischer (Ksiega Rodzaju) bzw. deutscher Sprache (Genesis), aber eben unter anderen Namen. Es ist auch ein Objekt christlicher Religion und damit $\mathrm{u}$. a. polnischer bzw. deutscher Kultur. Das Universalwörterbuch Duden kennt das Wort Genesis (1. Schöpfungsgeschichte, 2.1. Buch Mose), nicht aber das Wort Bereschit. Ist das Wort Tora die Bezeichnung einer Realie? Zwar werden so die 5 Bücher Mose im Judentum (im Christentum Pentateuch. Pl. Pięcioksiag) genannt, die Christen nennen aber diese Bücher nicht Tora. Darüber hinaus bedeutet Tora viel mehr und ist als grundlegender Begriff des Judentums ein eindeutiger Hinweis auf das Jüdische (Identitätsträger).

Im Grunde genommen hat eine Klassifikation der Bezeichnungen wie Tora oder Bereschit als Realien, Fremdwörter (im polnischen Fremdwörterbuch von Kopaliński ist Tora als Stichwort verzeichnet, Talmud übrigens auch; nicht aber das Wort Bereszit) oder Fachwörter für einen Übersetzungsprozess eine eher zweitrangige Bedeutung13. Die Hauptsache ist, wie sie übersetzt werden. In Bezug auf Tora war die Sache eindeutig. Unabhängig von jeglicher Klassifizierung als Fachwort, Fremdwort, Eigen- bzw. Fremdbezeichnung, funktioniert das Wort in beiden Sprachen und braucht keine Explikation. Ähnlich wie Talmud oder Rabbi u. a. Etwas anders verhält es sich mit dem Wort Bereschit. Wenn man es ebenfalls als Zitatwort übernimmt, braucht es noch zusätzlich eine Explikation, dass darunter das 1. Buch Mose $\mathrm{zu}$ verstehen ist. Es unterliegt keinem Zweifel, dass beide Bezeichnungen sowohl dem AS- als auch dem ZS-Text eine bestimmte - sozusagen eine jüdische - Färbung verleihen. Dies ist ein Mehrwert, ein stilistisches Potenzial von Realienbezeichnungen, auch wenn es nur scheinbare Realien sind. Da sie keine Entsprechungen in der Zielsprache haben, werden sie oft als Zitatwörter übernommen (vgl. Koller 1997; Markstein 1999). So war es auch im hier analysierten Text. Anzumerken ist jedoch, dass das dabei vorherrschende Übersetzungsverfahren nicht als reine Übernahme (im Sinne von Koller (1997), der dieses Verfahren auch als lexikalische Entlehnung bezeichnet $\left.^{14}\right)$ stattfand, sondern als Übernahme samt Explikation, also eher als Expandieren (im Sinne von Kautz 2000) realisiert wurde.

Wohlgemerkt, der AS-Text enthält meistens keine Erläuterungen bzw. erklärenden Hinweise oder Zusätze zu verwendeten Bezeichnungen. Und so lesen wir im AS-Text z.B.:

[1] Bożych ludzi czasami porywa Szechina $i$ ida przed siebie, tam gdzie stychać jej wotanie. Oder:

${ }^{13}$ Anders ist es in der Lexikologie oder Fachtextlinguistik der Fall.

${ }^{14}$ Vgl. dazu Schreiber (1999: 152). 
[2] Zdjęci groza Jamim Noraim, poruszeni natchnionymi mowami rebego, wszyscy wpadli w religijne uniesienie, wznoszac swoje żarliwe modty, przepraszajac i wybaczając sobie nawzajem.

Szechina und Jamim Noraim sind nicht die einzigen Beispiele für rätselhafte Bezeichnungen im AS-Text. Ohne explizite Erläuterungen kommen viele weitere Bezeichnungen vor, wie z.B. jorcajt, kraje galutu, chanukija, Erec Israel, Tyszebow, aron ha-kodesz, mizrach, Ezrat Gwarim, maariw u. v. a. m. Bei der Übersetzung ins Deutsche hielten wir es dennoch für angemessen, die meisten exotischen kulturspezifischen Bezeichnungen zu explizieren, da sie sonst für einen durchschnittlichen deutschen Leser unverständlich sind. Auch umgekehrt verstehen die AS-Leser die meisten im AS-Text verwendeten Bezeichnungen nicht. Dies liegt aber nicht mehr an der Kompetenz des Übersetzers, sondern an der des Autors bzw. des Interviewten. Im ZS-Text ist daher zu lesen:

[1'] Gottesmenschen werden manchmal von der Schechina, der Gegenwart Gottes in der Welt, hingerissen und sie begeben sich dorthin, wohin ihre innere Stimme sie ruft.

[2'] Während dieser Jamim Noraim, der ehrfurchtgebietenden Tage, und von den inspirierenden Reden des Rabbiners ergriffen, sind sie in eine religiöse Ekstase gefallen, indem sie eifrig gebetet und sich gegenseitig um Vergebung gebeten haben.

Auf solche expandierende Art und Weise (Übernahme + Explikation) wurden alle kultur- und fachspezifischen Bezeichnungen übersetzt, ungeachtet dessen, dass im AS-Text direkte Explikationen fehlten. Stattdessen gab es im AS-Text ziemlich oft, aber nicht immer, latente Hinweise, die samt Kontext und Kotext das Verstehen erleichterten, aber es nicht garantierten, z.B.

[3] Kilka miesięcy temu, grupa ortodoksyjnych chasydów z Jerozolimy zajechała do synagogi na wieczorne maariw.

Im AS-Text geben das Adjektiv wieczorne sowie der Kontext und Kotext Hinweise darauf, dass es beim Wort Maariw / Maariv vermutlich um eine Art Andacht / Gebet geht. Im ZS-Text wird dies durch den Zusatz Abendandacht eindeutig expliziert.

[3'] Vor einigen Monaten ist in die Synagoge eine Gruppe von orthodoxen Juden aus Jerusalem zur Abendandacht - Maariv angekommen.

Die exotischen kulturspezifischen Bezeichnungen und ihr Beibehalten im ZS-Text bei gleichzeitiger Gewährleistung, dass sie verständlich sind, waren aus den bereits genannten Gründen wichtig. Diese Bezeichnungen sind nicht nur ein textueller Beweis für Authentizität und Glaubwürdigkeit des Textes, sondern auch eine Brücke zwischen den drei Kulturen, und ein Hinweis auf Spuren der jüdischen Welt heute. 


\subsection{Kulturspezifische exotische Bezeichnungen und ihre Wiedergabe im ZS-Text}

Aus obigen Ausführungen geht hervor, dass nicht alle im Text vorkommenden fremden Bezeichnungen als Realien klassifiziert werden können. Deswegen wird im Weiteren von kulturspezifischen exotischen Bezeichnungen die Rede sein.

Im analysierten Text können zunächst zweierlei Arten von kulturspezifischen Bezeichnungen unterschieden werden, nämlich Bezeichnungen, die auf die polnische Kultur (grundsätzlich alle Toponyme und Eigennamen) und Bezeichnungen, die auf die jüdische Kultur hindeuten. Gemeint sind damit alle Bezeichnungen und Wendungen, die entweder aus dem Hebräischen oder dem Jiddischen übernommen wurden.

Innerhalb der zweitgenannten Gruppe lassen sich Untergruppen von Bezeichnungen differenzieren, je nach Lebensbereich, auf den sich die jeweilige Bezeichnung bezieht. Die nachstehend angebrachten Beispiele zeigen ausgewählte Bezeichnungen in polnischer und deutscher Sprache samt Explikation, wobei sie grob in zwei grundsätzliche Bereiche unterteilt wurden: Bezeichnungen verbunden mit dem Alltag und Bezeichnungen verbunden mit Festen, Feiern, Glauben, Religion:

Tabelle 1. Beispiele für kulturspezifische exotische Bezeichnungen aus dem Hebräischen und dem Jiddischen, verbunden mit Festen, Feiern, Glauben, Religion.

\begin{tabular}{|c|c|c|}
\hline Polnisch & Deutsch & $\begin{array}{l}\text { Explikation (die meist im ZS-Text samt } \\
\text { Zitatwort erscheint) }\end{array}$ \\
\hline \multicolumn{3}{|c|}{ Bezeichnungen für Feste } \\
\hline Jom Kippur & Jom Kippur & das höchste jüdische Fest \\
\hline Jamim Noraim & Jamim Noraim & ehrfurchtgebietende Tage \\
\hline jorcajt & Jorcajt & Todestag (Jahrzeit $\left.{ }^{* *}\right)$ \\
\hline \multicolumn{3}{|c|}{$\begin{array}{l}\text { Bezeichnungen verbunden mit Religion und Glauben, religiösen Riten, profanen Sitten } \\
\text { und Gebräuchen }\end{array}$} \\
\hline micwa & Mitzwa & Gebot \\
\hline macewa & Matzewa/Matzeva & Grabstein \\
\hline cadyk & Zaddik Pl. Zaddikim & [als heilig verehrter] Lehrer im Chassidismus ${ }^{* *}$ \\
\hline chasyd & Chassid, Pl. Chassidim & der Fromme $e^{* *}$ \\
\hline rabin & Rabbiner & Schriftgelehrter / Religionslehrer** \\
\hline maariw & Maariw/Maariv & Abendandacht / Abendgebet \\
\hline kwitłech & Kwitlech & $\begin{array}{l}\text { Fürbittezettel / Papierschnipsel mit Bitten } \\
\text { um Fürsprache bei Gott }\end{array}$ \\
\hline
\end{tabular}




\begin{tabular}{|c|c|c|}
\hline Polnisch & Deutsch & $\begin{array}{l}\text { Explikation (die meist im ZS-Text samt } \\
\text { Zitatwort erscheint) }\end{array}$ \\
\hline \multicolumn{3}{|c|}{ Bezeichnungen von Gegenständen, die man an Feiertagen (aber auch im Alltag) benutzte } \\
\hline chałat & - & chassidischer Mantel \\
\hline talit & Tallit & Gebetsmantel \\
\hline filakteria / tefilin & Tefillin (seltener Phylakterie)** & Gebetsriemen / Gebetskapseln \\
\hline machzor & Machsor Pl. Machsorim & Gebetbuch (für Festtage ${ }^{* *}$ ) \\
\hline sidur & Sidur Pl. Sidurim & Gebetbuch (für den Alltag**) \\
\hline chanukija & Chanukkaleuchter & \\
\hline \multicolumn{3}{|c|}{ Bezeichnungen für Einrichtung und Ausstattung der Synagoge } \\
\hline aron ha kodesh & Aron Ha Kodesh & der heilige Schrein \\
\hline mezuza & Mesuse, Pl. Mesusot & Schriftkapseln an Türpfosten ${ }^{* * *}$ \\
\hline babiniec & & Frauenempore bzw. Frauengebetsraum \\
\hline geniza & Genisa (auch Geniza) & Lager, Depot, Speicher** \\
\hline bima & Bima & $\begin{array}{l}\text { Pult od. Podium - der Platz in der Synagoge, } \\
\text { von dem aus die Tora gelesen wird. }{ }^{* *}\end{array}$ \\
\hline Ezrat Gwarim & Ezrat Gwarim & der große Gebetraum für Männer \\
\hline Tora & Tora & - \\
\hline Bereszit & Bereschit & 1. Buch Mose (Genesis**) \\
\hline Szechina & Schechina & $\begin{array}{l}\text { Inbegriff der Gegenwart Gottes bei seinem } \\
\text { Volk }\end{array}$ \\
\hline
\end{tabular}

* In diesem Fall enthält auch der AS-Text Explikationen, wofür der jeweilige Name steht.

** Die kursiv gedruckten Explikationen erscheinen im ZS-Text nicht.

*** Mesusa ist nicht nur ein Ausrüstungsstück einer Synagoge, sondern auch jedes jüdischen Hauses.

Die Suche nach deutschen Äquivalenten war nicht immer erfolgreich. Nehmen wir als Beispiel das Wort chałat. Die polnische Bezeichnung chatat bedeutet 1 . «długie okrycie wierzchnie przypominające płaszcz, noszone zwłaszcza przez Żydów we wschodniej Europie» 2. pot. «luźne okrycie, np. fartuch ochronny lub szlafrok szpitalny» 3. pot. «o zniszczonym lub zbyt obszernym ubraniu» (vgl. http://sjp.pwn.pl/sjp/chalat;2553257.html). Die 1. Lesart ist eine Realienbezeichnung. Die zwei übrigen sind keine Realienbezeichnungen mehr. Das Wort chatat funktioniert im Polnischen auch als gewöhnliches Lexem der Alltagssprache (die Lesart 3). Die Suche nach einer Realienbezeichnung im Deutschen blieb erfolglos, deswegen erscheint im ZS-Text die explikative Analogiebildung chassidischer Mantel.

Der AS-Text enthält manchmal idiolektale autorenspezifische Bezeichnungen, z.B. wird das Wort chanukija im AS-Text als Zitatwort aus dem Heb- 
räischem verwendet. Im Polnischen sind chanukowy świecznik oder chanukowa lampa, manchmal auch chanuka, gebräuchlicher ${ }^{15}$.

Nicht immer war die Ermittlung des Inhalts einer Bezeichnung einfach und eindeutig. Schon gar nicht eindeutig war die Schreibweise. Viele Bezeichnungen und Namen erwiesen sich als Bezeichnungen mit instabiler Schreibweise. Je nach Quelle findet man bei der Recherche verschiedene transkribierte Varianten. Weltweit gibt es viele Transkriptionen, die für das Hebräische verwendet werden, $u$. a. die DIN 31636. Der AS-Text ist aber kein Informationstext. Er ist ein Interview, in dem eine ehemalige galizische und jüdische Lebenswelt wiederbelebt wird. Aus seinen „Ungenauigkeiten“ lässt

Tabelle 2. Beispiele für kulturspezifische exotische Bezeichnungen aus dem Hebräischen und dem Jiddischen, verbunden mit dem Alltag.

\begin{tabular}{|c|c|c|}
\hline \multicolumn{3}{|c|}{ Historische Ereignisse } \\
\hline Schoah & Schoah & - \\
\hline \multicolumn{3}{|c|}{ Datenangaben } \\
\hline Tyszebow & Tische beAv & $\begin{array}{l}\text { Gedenktag der Zerstörung des } \\
\text { ersten und des zweiten Tempels } \\
\text { in Jerusalem }\end{array}$ \\
\hline \multicolumn{3}{|c|}{$\begin{array}{c}\text { Bezeichnungen von Personen (Funktionen, Eigenschaften, Namen/Beinamen, fiktiven } \\
\text { Gestalten/Figuren) }\end{array}$} \\
\hline Szojmer & Schojmer & Aufpasser, Wächter \\
\hline Chojze & Chozeh & der Seher \\
\hline Tewje mleczarz & Tewje Milchmann & - \\
\hline \multicolumn{3}{|c|}{ Toponyme/ Lokale Bezeichnungen } \\
\hline kraje galutu & Galutstaaten & die jüdische Diaspora \\
\hline Eden & Garten Eden & - \\
\hline Erec Israel & Eretz Israel & das Heilige Land \\
\hline Polin & Po-lin & Polen \\
\hline \multicolumn{3}{|c|}{ Bezeichnungen von Institutionen, Behörden und Organisationen* } \\
\hline Dom Ludowy Bejt ha-Am & $\begin{array}{l}\text { das jüdische Volkshaus Bejt } \\
\text { ha-Am }\end{array}$ & - \\
\hline Orkiestra Ha-Zamir & das Orchester Ha-Zamir & - \\
\hline $\begin{array}{l}\text { Ortodoksyjne Machzikej } \\
\text { ha-Dat }\end{array}$ & $\begin{array}{l}\text { die orthodoxe Organisation } \\
\text { Machzikej ha-Dat }\end{array}$ & - \\
\hline Chewra Kadisza & Chewra Kadischa & Beerdigungsgesellschaft \\
\hline
\end{tabular}

* In diesem Fall enthält auch der AS-Text Explikationen, wofür der jeweilige Name steht.

** Die kursiv gedruckten Explikationen erscheinen im ZS-Text nicht.

${ }^{15}$ Vgl. http://www.jhi.pl/psj/chanukowy_swiecznik. (Letzter Zugriff am 14.08.2016). 
sich seine Authentizität erschließen. Deswegen galt als Faustregel bei der Übersetzung, dass wir diese Bezeichnungen in der Schreibweise des AS-Textes belassen. So steht im ZS-Text das Wort Schojmer. Erst im Nachhinein stellte sich heraus, dass im Deutschen das Wort Hashomer hierfür geeigneter wäre ${ }^{16}$.

Die obigen Belege bringen deutlich zum Vorschein, dass sich die meisten Bezeichnungen als Zitatwörter übersetzen lassen. Diese Zitatwörter funktionieren in beiden Sprachen, auch wenn es zwischen den jeweiligen Äquivalenten geringe Unterschiede in der Schreibung gibt (z.B. Pl. mezuza, Dt. Mesusa ${ }^{17}$ ). Bemerkenswert ist in diesem Kontext, dass diese Wörter im Deutschen eher wie Fremdwörter funktionieren und die Pluralformen gemäß dem Hebräischen gebildet werden (vgl. Zaddikim, Chassidim, Mesusot). Im Polnischen funktionieren sie wie Lehnwörter. Die Pluralformen werden gemäß polnischer Grammatikregeln gebildet (vgl. cadykowie, chasydzi, mezuzy). Zur Hilfe bei der Übersetzung kam hier der Duden, in dem die meisten Pluralformen nachzuschlagen sind. Bei manchen sind zwei Varianten angegeben: Chassid, Pl. Chassidim auch Chassiden, aber Zaddik, Pl. Zaddikimis.

Innerhalb dieser Gruppe kann eine Untergruppe ausgesondert werden, die diejenigen Wörter bilden, die bei der Übersetzung im ZS-Text ohne Explikation auskommen, z.B. Tora, Talmud, Shoah, Bima, Genisa. Bis auf Bima und Genisa kann vorausgesetzt werden, dass der Bekanntheitsgrad dieser Wörter unter Lesern ausreichend ist, daher sind keine zusätzlichen Erläuterungen nötig. Sie funktionieren in der jeweiligen Sprache wie andere Fachbezeichnungen, Eigennamen, Fremdwörter, Internationalismen und sind in Nachschlagewerken, z. B. im Duden, erfasst.

Unter den angeführten Beispielen gibt es auch einige wenige, die ins Polnische als Zitatwörter übernommen und ins Deutsche mit Hilfe eines lexikalisch nahen Wortes durch Umschreibung übertragen wurden ${ }^{19}$ : Pl. chatat anders kapota genannt ${ }^{20}$ vs. Dt. chassidischer Mantel. Zwischen der AS-Bezeichnung und der ZS-Bezeichnung gibt es dann eine andere Art Äquivalenz als z. B. innerhalb des Paars: Pl. mezuza - Dt. Mesusa. Es lassen sich aber auch

${ }^{16}$ Hashomer (hebr. רמושה) bedeutet Wache, Wächter, z B. Hashomer Hazair (eine internationale pfadfinderähnliche Organisation). Zur Zeit der Übersetzung war dies uns nicht bekannt, deswegen steht im ZS-Text: Schojmer.

17 Aber auch Mezuzah oder Mesusah, Plural: Mesusot (vgl. https://de.wikipedia.org/wiki/ Mesusa).

18 Vgl. http://www.duden.de/rechtschreibung/Chassid und http://www.duden.de/su chen/dudenonline/Zaddik (letzter Zugriff am 13.08.2016).

${ }_{19}$ Markstein nennt dieses Übersetzungsverfahren Analogiebildung (vgl. Markstein 1999: 291).

${ }^{20}$ Chatat (arabisch: chilat; türkisch: chalat) auch kapota (jiddisch: kapote) gennant. (Vgl. http://www.jhi.pl/psj/chalat; letzter Zugriff am 13.08.2016). 
Paare von Bezeichnungen aufzeigen, wo sowohl die AS-Bezeichnung als auch die ZS-Bezeichnung in Form eines Zitatwortes zusammen mit einer Analogiebildung funktioniert, z. B. Pl. chanukowa lampa (chanukowy świecznik) - Dt. Chanukkaleuchter.

Eine andere Gruppe der obigen Bezeichnungen stellen diejenigen dar, die sowohl im AS- als auch im ZS-Text samt Explikation vorkommen. Bezeichnungen, wie z. B. Bejt Midrasz / Beit Midrasch oder Chewra Kadischa sind wirklich nur für eingeweihte Leser des AS- bzw. ZS-Textes verständlich, daher ist ihre Verwendung ohne gleichzeitige Erklärung wenig sinnvoll. Es sind meistens hebräische Eigennamen ${ }^{21}$ verschiedener Organisationen, Gesellschaften und Einrichtungen (also auch Realienbezeichnungen).

Zur Übersetzung von Realienbezeichnungen könnte man noch Bände schreiben, aber im Kontext dieses Beitrags ist der übersetzungsdidaktische Aspekt besonders relevant. Die Projektteilnehmer konnten zielgerichtete Internetrecherche praktizieren und verschiedene Übersetzungsverfahren ausprobieren. Sie sind sich auch bestimmter Unterschiede in Bezug auf das Funktionieren von (scheinbaren) Realienbezeichnungen in beiden Sprachen bewusst geworden. Z.B. sind manche im Polnischen eingebürgerte Realien dem Deutschen völlig fremd (Pl. chałat) bzw. weitgehend fremd (Pluralformen: Dt. Zaddikim, Chassidim). Wichtig war dabei auch die Erkenntnis, dass man sich als Übersetzer oft mit bestimmten Kompromisslösungen zufrieden geben muss und dass Übersetzen die Qual der Wahl bedeutet, wobei eben "die Wahl“ nicht immer das ist, was alle Facetten widerspiegelt. Für die jüngsten unter uns war es sicher auch eine Art Entdeckung, dass nicht alles in Wörterbüchern und Lexika zu finden ist und dass man als Übersetzer oft entscheiden muss, welche von den zur Verfügung stehenden „Halblösungen“ man wählt und warum (!). Das „Warum“ will nämlich begründet sein - und das bedeutet ständiges (Nach)Grübeln.

\section{SCHLUSSBEMERKUNGEN}

Dank ihres Engagements haben die Projektteilnehmer bestimmt viel Neues sowohl in Bezug auf ihre Sprachkenntnisse als auch übersetzerische Kompetenz gelernt. Dazu kommt noch eine Erweiterung ihres Allgemeinund Fachwissens und Übungen in der Teamarbeit, Internet- und Quellenrecherche. Außer den genannten Erkenntnissen ist auch der erzieherische und kulturelle Aspekt wichtig. Die Arbeit an diesem Text animierte die Teilnehmer dazu, sich die frühere materielle Existenz der Juden vor Augen zu

${ }^{21}$ Allerdings steht Chewra Kadisza im AS-Text ohne Erläuterung. 
führen und in diesem Kontext sich vielleicht die Fragen zu stellen: Wie ist meine Einstellung der beschriebenen Problematik gegenüber? Die Synagoge in Łańcut wurde für manche Projektteilnehmer zu einem wichtigen Ort, den man anderen zeigen und erläutern will.

Die Teamarbeit bedeutete eine Zusammenarbeit mit anderen und ein gemeinsames Redigieren der Endfassung, sie schloss aber die individuelle Verantwortung nicht aus: Leistungen des Einzelnen wurden meistens im Plenum ausgewertet. Der Übersetzer musste seine Entscheidungen begründen können. Nicht zu unterschätzen war in diesem Kontext die muttersprachliche Unterstützung und die Nachbearbeitung des Textes durch den Muttersprachler. Seine Anwesenheit und Hilfsbereitschaft waren außer den sich aus der authentischen Situation und einem echten Übersetzungsauftrag ergebenden Motivationsverstärkern natürliche Anspornmechanismen für die Projektteilnehmer.

Und last but not least, das Projekt, dessen Teilnehmer den zu übersetzenden Text aus verschiedenen Blickwinkeln zu erfassen hatten (ihre eigene Perspektive, die Perspektive des Übersetzers, die des Muttersprachlers) war somit auch ein Lehr- und Lernangebot in ethischer Hinsicht: Denn eine Übersetzung bedarf außer sprachlicher Korrektheit und semantischer Äquivalenz noch loyal zu sein. Nord brachte dies so auf den Punkt: „Es geht also beim Übersetzen nicht um einen absoluten Begriff der "Treue" $\mathrm{zu}$ einem Text (!), sondern um verschiedene Möglichkeiten der Herstellung funktionsgerechter Translate im Rahmen des (ethischen) Prinzips der Loyalität gegenüber den beteiligten Partnern." (Nord 2011: 27). Diese Partner sind v. a. der Autor, (der Auftraggeber), und der Empfänger des Textes, aber auch der Übersetzer selbst.

\section{LITERATURVERZEICHNIS}

Burszta, W. (1998). Antropologia kultury. Tematy, teorie, interpretacje. Poznań: Wydawnictwo Zysk i S-ka.

Kautz, U. (2000). Handbuch Didaktik des Übersetzens und Dolmetschens. München: Iudicium Verlag.

Koller, W. (1997). Einführung in die Übersetzungswissenschaft. Wiesbaden: Quelle \& Meyer.

Markstein, E. (1999). Realia. In: M. Snell-Hornby / H. G. Hönig / P. Kußmaul / P. A. Schmitt (Hrsg.), Handbuch Translation (S. 288-291). Tübingen: Stauffenburg Verlag.

Nord, C. (1999). Ausrichtung an der zielkulturellen Situation (S. 144-147). In: M. Snell-Hornby / H. G. Hönig / P. Kußmaul / P.A. Schmitt (Hrsg.), Handbuch Translation. Tübingen: Stauffenburg Verlag.

Nord, C. (2011). Funktionsgerechtigkeit und Loyalität: Theorie, Methode und Didaktik des funktionalen Übersetzens. Berlin: Frank \& Timme GmbH.

Potocki, A. (2004). Żydzi w Podkarpackiem. Rzeszów: Libra. 
Schreiber, M. (1999). Übersetzungstypen und Übersetzungsverfahren (S. 151-154). In: M. SnellHornby / H. G. Hönig / P. Kußmaul / P.A. Schmitt (Hrsg.), Handbuch Translation. Tübingen: Stauffenburg Verlag.

Internet-Quellen:

Deutsches Wörterbuch von Jacob Grimm und Wilhelm Grimm: http:// woerterbuchnetz.de/DWB

Forum Żydów Polskich: http://www.fzp.net.pl

Fundacja Ochrony Dziedzictwa Żydowskiego: http:// fodz.pl

http://www.duden.de

https://de.glosbe.com/pl/de/

Polski Stownik Judaistyczny: www.jhi.pl/psj

Rosnacy stownik hebrajski: http://www.iwrit.pl/index.php?ww=1\&slowo=nie\&ccnb=on

Stownik jezzyka polskiego: http://sjp.pwn.pl

Received: 5.06.2017; revised: 27.09.2017 